Received: 2016.03 .27

Accepted: 2016.06.07

Published: 2016.07.11

Authors' Contribution:

Study Design A

Data Collection B

Statistical Analysis C

Data Interpretation D

Manuscript Preparation E

Literature Search F

Funds Collection G

Corresponding Author:

Source of support:

Background:

Material/Methods:

Results:

Conclusions:

MeSH Keywords:

Intestinal Neoplasms • MicroRNAs • Real-Time Polymerase Chain Reaction

Full-text PDF:

\author{
BCDE Deng-Rui Liu \\ ACFG Quan-Lin Guan \\ BCDE Ming-Tai Gao \\ CEF Lei Jiang \\ BEF Hong-Xia Kang
}

Quan-Lin Guan, e-mail: gansugql@163.com

This work was supported by grants from the Natural Science Foundation of Gansu Province (number 145RJZA229) of miR-1260b abnormal expression with clinical pathological features in CRC patients. vival for CRC patients. poor prognosis of patients with CRC. nosis for patients with CRC.

http://www.medscimonit.com/abstract/index/idArt/898733

\title{
miR-1260b is a Potential Prognostic Biomarker in Colorectal Cancer
}

Department of Surgery, The First Hospital of Lanzhou University, Lanzhou, Gansu, P.R. China

Colorectal cancer (CRC) mainly refers to colon and rectum cancer, which is the most common gastrointestinal malignant tumor. MicroRNAs (miRNAs) in tumors participate in multiple processes of malignancy development, including cell differentiation, proliferation, invasion, and metastasis. In this study we explored the relationship

The expression of miR-1260b was detected by real-time quantitative polymerase chain reaction (real-time PCR) in 120 cases of CRC tissues. The correlation of miR-1260b expression with the clinicopathologic features of CRC was analyzed by SPSS 21.0 statistical software. The Kaplan-Meier method was used for survival analysis. Cox regression analyses were conducted to determine whether miR-1260 b was an independent predictor of sur-

The miR-1260b expression in CRC was significantly higher than the expression levels in the corresponding para-carcinoma tissues $(P<0.001)$. According to the expression levels of miR-1260b, 120 cases of CRC patients were classified into either the miR-1260b high expression group or the miR-1260b low expression group. The high expression levels of miR-1260b in CRC patients was associated with lymph node metastasis $(P<0.05)$ and venous invasion $(P<0.001)$. However, the high miR-1260b expression had no significant correlation with other clinical parameters $(P>0.05)$. The high miR-1260b expression patients survived for shorter times than those CRC patients with low miR-1260b expression $(P<0.05)$. Multivariate analysis revealed that high miR-1260b means

The high expression level of miR-1260b is an independent prognostic biomarker that indicates a worse prog-

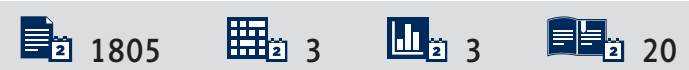




\section{Background}

Colorectal cancer (CRC) mainly refers to colon and rectum cancer, which is the most common gastrointestinal malignant tumor. The incidence and mortality rates of CRC are next only to those of gastric cancer, esophageal cancer, and primary liver cancer, which are all cancers that afflict the digestive system [1]. CRC patients at stage I-II and stage III have a better prognosis and five-year survival rate is $60-80 \%$. However, prognosis of advanced CRC and postoperative recurrence of CRC is poor [2]. A large number of molecules and complicated signaling pathway regulations are involved in the occurrence and development of $C R C$, among which are oncogene activation, tumor suppressor gene inactivation, and epigenetic modifications [3]. Currently, research effort is being made to elucidate CRC pathogenesis, and there is an interest in further exploring the molecular dynamics and to target the molecular abnormalities in CRC.

MicroRNAs (miRNAs) are small RNAs (19-24 nucleotides in length) encoded by endogenous genes. miRNAs complementarily bind to the 3' untranslated regions (UTRs) of target genes to form the RNA-induced silencing complex (RISC), which results in the degradation of mRNA and inhibits the expression of target genes by translation or other regulatory mechanisms $[4,5]$. miRNAs can participate in many biological activities, including cell division, hormone secretion, and cancer development and progression [6]. More than 1000 kinds of human miRNA have been identified, and over $50 \%$ of the miRNA genes are located at susceptibility loci of tumor-associated chromosomes [7,8]. Compared with normal tissues, miRNA expression in tumor tissues is often significantly up-regulated or down-regulated. Such expression patterns act similarly to oncogenes or tumor suppressor genes by regulating the

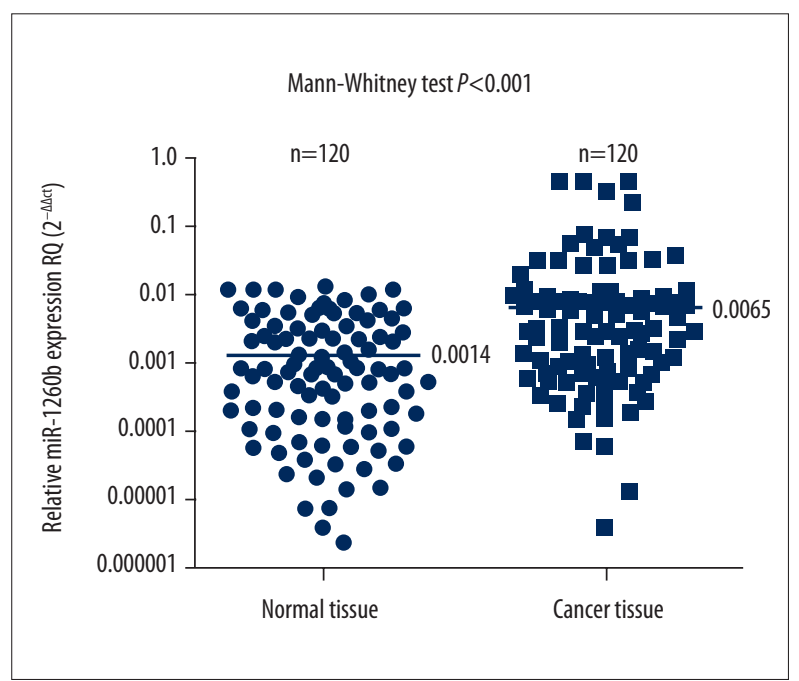

Figure 1. Expression level of miR-1260b in colorectal cancer and normal tissues. downstream tumor-associated genes. Thus, miRNAs in tumors participate in multiple processes of malignancy development, including cell differentiation, proliferation, invasion, and metastasis $[9,10]$. By reviewing the relevant literature, we found that miR-1260b is abnormally expressed in multiple tumors, indicating that it may be a carcinogenic or tumor-suppressive miRNA. At present, the expression of miR-1260b in CRC is unclear. In the present study we investigated the expression levels of miR-1260b in CRC tissues and para-carcinoma tissues by real-time quantitative polymerase chain reaction (real-time PCR). We also explored the relationship of miR-1260b abnormal expression with clinical pathological features in CRC patients.

\section{Material and Methods}

\section{Patients and samples}

CRC tissues and adjacent tissues were obtained from 120 patients who had undergone complete surgical resection at the First Hospital of Lanzhou University from January 2009 to October 2010. All patients were confirmed for CRC by pathology, and they did not receive chemotherapy, radiotherapy, or immunotherapy before surgery. According to the TNM staging system developed by the American Joint Committee on Cancer (AJCC)/Union for International Cancer Control (UICC), clinical staging for patients was performed. Specifically, 34 cases were in the I stage, 55 cases were in the II stage, and 31 cases were in the III stage. In this study, all patients with complete clinicopathological data signed an informed consent form. This investigation was approved by the in-house Medical Ethics Committee of the First Hospital of Lanzhou University.

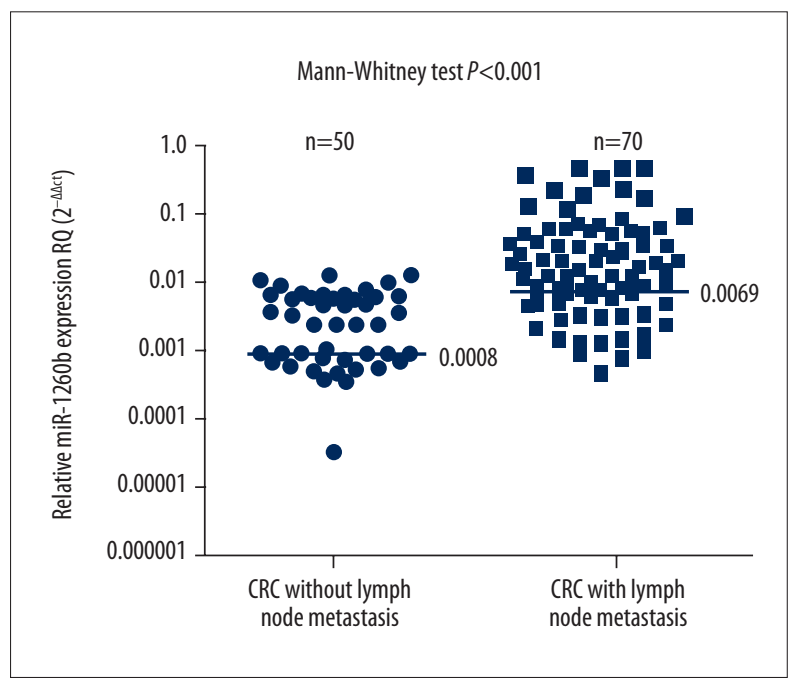

Figure 2. Expression level of miR-1260b in colorectal cancer tissues with and without lymph node metastasis. 
Table 1. Correlation between miR-1260b expression and clinicopathological factors of colorectal cancer.

\begin{tabular}{|c|c|c|c|}
\hline \multirow{2}{*}{ Variable } & \multicolumn{2}{|c|}{ miR-1260b expression } & \multirow{2}{*}{$P$ value } \\
\hline & Low $(n=53)$ & High $(n=67)$ & \\
\hline Age (yr) & & & 0.437 \\
\hline$\leq 60$ & 33 & 37 & \\
\hline$>60$ & 20 & 30 & \\
\hline Gender & & & 0.880 \\
\hline Male & 30 & 37 & \\
\hline Female & 23 & 30 & \\
\hline Tumor size & & & 0.888 \\
\hline$\leq 5 \mathrm{~cm}$ & 26 & 32 & \\
\hline$>5 \mathrm{~cm}$ & 27 & 35 & \\
\hline Degree of differentiation & & & 0.509 \\
\hline Well and moderate & 35 & 48 & \\
\hline Poor & 18 & 19 & \\
\hline Venous invasion & & & $<0.001$ \\
\hline Negative & 50 & 45 & \\
\hline Positive & 3 & 22 & \\
\hline Depth of invasion & & & 0.091 \\
\hline $\mathrm{T} 1, \mathrm{~T} 2$ & 22 & 18 & \\
\hline $\mathrm{T} 3, \mathrm{~T} 4$ & 31 & 49 & \\
\hline Lymph node metastasis & & & 0.027 \\
\hline Absent & 28 & 22 & \\
\hline Present & 25 & 45 & \\
\hline Location & & & 0.821 \\
\hline Colon & 25 & 33 & \\
\hline Rectum & 28 & 34 & \\
\hline
\end{tabular}

\section{Real-time PCR}

CRC tissues and adjacent tissues were extracted for miR-1260b. Trizol (Invitrogen, CA) was used to extract the total RNA and was conducted as per the manufacturer's instructions. Total RNA isolated from patient samples were eluted in $50 \mu \mathrm{L}$ diethyl pyrocarbonate (DEPC) water and stored at $-80^{\circ} \mathrm{C}$ until use. The total RNA concentration was measured by ultraviolet spectrophotometry. Reverse transcription was carried out using reverse transcription kits (RR037A, TaKaRa Company, Japan) with $0.5 \mu \mathrm{g}$ RNA, totaling to a final volume of $20 \mu \mathrm{L}$. Other reagents included: $5 \mu \mathrm{L} 5 \times$ PrimeScript Buffer, $1 \mu \mathrm{L}$ PrimeScript RT Enzyme Mix I (Haoqin Biotechnology, Shanghai, China), $2 \mu \mathrm{L}$ stem-loop RT primer, and DEPC water. Reverse transcription reactions were as follows: $42^{\circ} \mathrm{C}$ for $15 \mathrm{~min}, 85^{\circ} \mathrm{C}$ for $5 \mathrm{~s}$, and $4^{\circ} \mathrm{C}$ for $15 \mathrm{~min}$, and then the reactions were stored at $-80^{\circ} \mathrm{C}$ until further use for real-time PCR.

Real-time PCR reagents included: $10 \mu \mathrm{L}$ Real-time PCR Master Mix, $1 \mu \mathrm{L}$ upstream primer, $1 \mu \mathrm{L}$ downstream primer, $2 \mu \mathrm{L}$ CDNA, and DEPC water to bring to a total volume of $20 \mu \mathrm{L}$. Real-time PCR was performed using the 7300 Real-Time PCR System (Applied Biosystems) under the following conditions: $95^{\circ} \mathrm{C}$ for $10 \mathrm{~min}$ and 40 cycles of $95^{\circ} \mathrm{C}$ for $15 \mathrm{~s}$ and $60^{\circ} \mathrm{C}$ for 1 min. Real-time PCR of patient samples were repeated 3 times. The expression levels of miR-1260b was calculated by the comparative cycle threshold (Ct) method, and U6 small nuclear RNA was used as an internal reference. The relative expression of miR-1260b to U6 was determined using the equation $2^{-\Delta \Delta c t}$, where $\Delta \Delta \mathrm{Ct}=\mathrm{Ct}_{\text {miR-1260b }}-\mathrm{Ct}_{\mathrm{U6}}[11]$. 

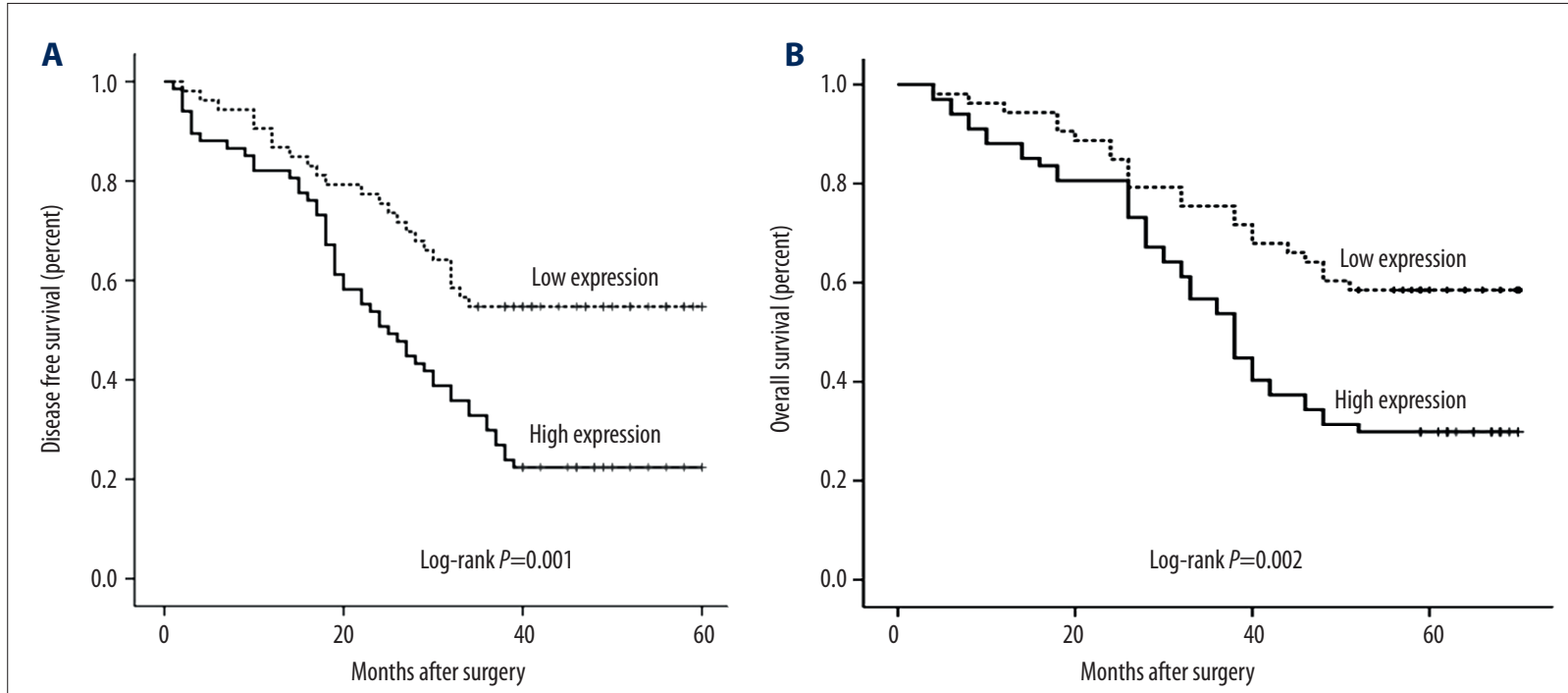

C

D
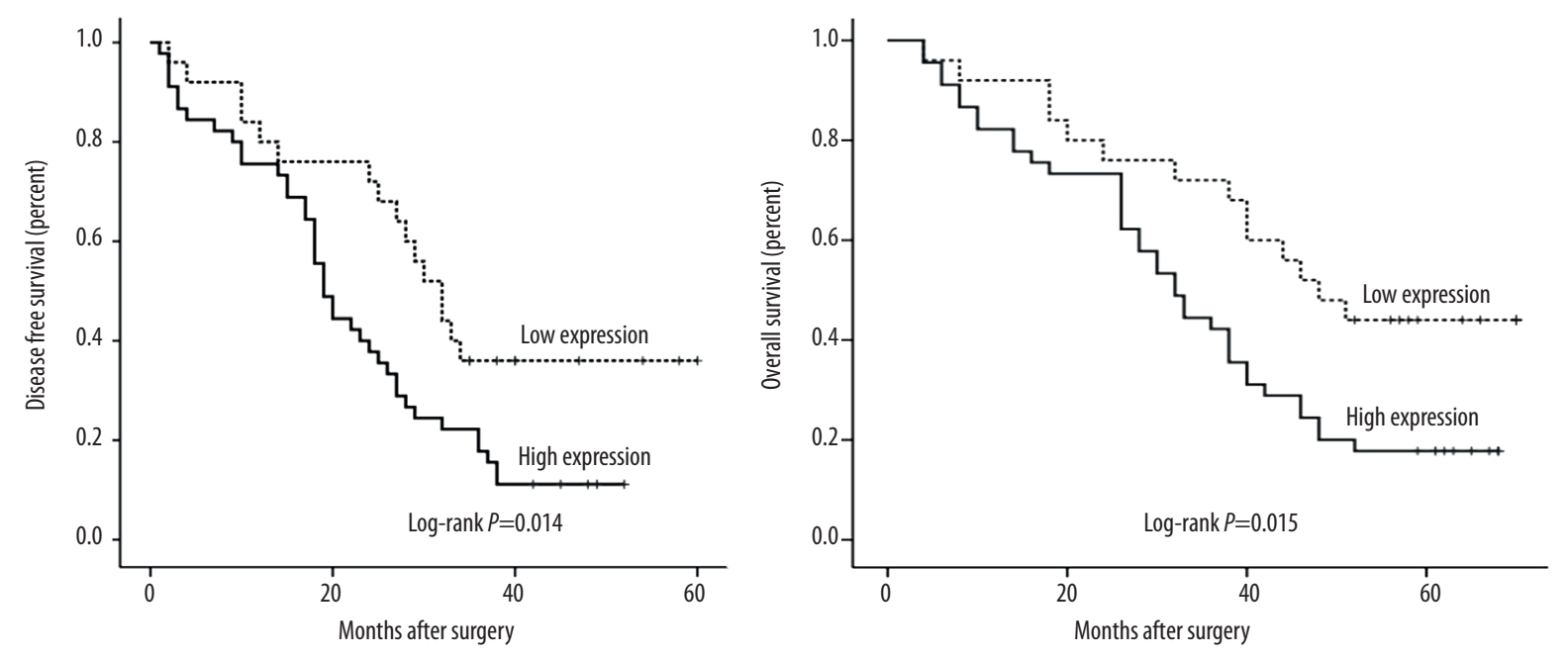

Figure 3. Kaplan-Meier survival curves of patients with colorectal cancer based on miR-1260b expression. (A) Disease-free survival of patients with colorectal cancer. (B) Overall survival of patients with colorectal cancer. (C) Disease-free survival of patients with lymph node metastases. (D) Overall survival of patients with lymph node metastases.

\section{Statistical methods}

We compared the miR-1260b expression in CRC cancer tissues with miR-1260b expression in para-carcinoma tissues or lymph node by paired t-test or Mann-Whitney test. Patients were divided into the miR-1260b low expression group and miR-1260b high expression group based on boundary values (median $2^{-\Delta \Delta c t}$ values). The chi-square test was used for miR$1260 \mathrm{~b}$ expression and clinicopathological parameters. The Kaplan-Meier method was used for survival analysis, and the log-rank test was used to compare patient survival between the 2 miR-1260b expression groups. To further analyze the survival data, the Cox proportional hazards model was used for joint effect analysis of each covariate. The overall survival
(OS) of CRC patients was considered as the primary outcome, and disease-free survival (DFS) was defined as the secondary outcome. Multivariate analysis was conducted using the Cox proportional hazards regression model. SPSS 21.0 statistical software (SPSS, Chicago, IL) was used for statistical analysis. $P$ value of $<0.05$ was regarded as statistically significant.

\section{Results}

\section{miR-1260b and CRC}

Of all patients, 67 were male and 53 were female. The age range was $37-78$ years old (average $53.6 \pm 10.2$ years old). Among the 
Table 2. Univariate analysis of clinicopathological factors for overall survival of colorectal cancer patients.

\begin{tabular}{|c|c|c|c|c|}
\hline Variable & Cases & HR & $95 \% \mathrm{Cl}$ & $P$ value \\
\hline Age (yr) & & & $0.604-1.571$ & 0.913 \\
\hline$\leq 60$ & 70 & 1 & & \\
\hline$>60$ & 50 & 0.974 & & \\
\hline Gender & & & $0.752-1.936$ & 0.436 \\
\hline Male & 67 & 1 & & \\
\hline Female & 53 & 1.207 & & \\
\hline Tumor size & & & $0.595-1.530$ & 0.847 \\
\hline$\leq 5 \mathrm{~cm}$ & 58 & 1 & & \\
\hline$>5 \mathrm{~cm}$ & 62 & 0.954 & & \\
\hline Degree of differentiation & & & $0.607-1.673$ & 0.976 \\
\hline Well and moderate & 83 & 1 & & \\
\hline Poor & 37 & 1.008 & & \\
\hline Venous invasion & & & $1.021-2.901$ & 0.041 \\
\hline Negative & 95 & 1 & & \\
\hline Positive & 25 & 1.721 & & \\
\hline Depth of invasion & & & $2.351-9.060$ & $<0.001$ \\
\hline $\mathrm{T} 1, \mathrm{~T} 2$ & 40 & 1 & & \\
\hline $\mathrm{T} 3, \mathrm{~T} 4$ & 80 & 4.615 & & \\
\hline Lymph node metastasis & & & $1.626-4.778$ & $<0.001$ \\
\hline Absent & 50 & 1 & & \\
\hline Present & 70 & 2.787 & & \\
\hline Location & & & $0.707-1.822$ & 0.600 \\
\hline Colon & 58 & 1 & & \\
\hline Rectum & 62 & 1.135 & & \\
\hline miR-1260b expression & & & $1.304-3.607$ & 0.003 \\
\hline Low & 53 & 1 & & \\
\hline High & 67 & 2.169 & & \\
\hline
\end{tabular}

120 patients in this study, there were 58 cases of colon cancer and 62 cases of rectal cancer. The miR-1260b expression in CRC was significantly higher than the expression levels in the corresponding para-carcinoma tissues $(P<0.001$, Figure 1$)$. To explore the relationship of miR-1260b expression level with lymph node status, the expression level of miR-1260b in patients with different lymph node status was analyzed. Compared with patients without lymph node metastasis, the miR-1260b expression levels increased significantly in CRC tissues with lymph node metastasis $(P<0.001)$ (Figure 2$)$.

\section{Expression of miR-1260b and clinicopathological features of CRC}

According to the expression levels of miR-1260b ( $2^{-\Delta \Delta \mathrm{ct}}$ median value as the cut-off value), 120 cases of CRC patients were classified into either the miR-1260b high expression group or the miR-1260b low expression group. Specifically, 67 patients with CRC were in the high expression group, and the other 53 were in the low expression group. We analyzed the relationship between clinicopathological features of CRC patients and miR-1260b expression (Table 1). The high expression levels of miR-1260b in CRC patients was associated with lymph node metastasis $(P<0.05)$ and venous invasion $(P<0.001)$. However, the high miR-1260b expression had no significant correlation with other clinical parameters, including age, sex, tumor 
Table 3. Multivariate analysis of clinicopathological factors for overall survival of colorectal cancer patients.

\begin{tabular}{|c|c|c|c|c|}
\hline Variable & Cases & HR & $95 \% \mathrm{Cl}$ & $P$ value \\
\hline Venous invasion & & & $0.630-2.011$ & 0.690 \\
\hline Negative & 95 & 1 & & \\
\hline Positive & 25 & 1.125 & & \\
\hline Depth of invasion & & & $2.138-8.759$ & $<0.001$ \\
\hline $\mathrm{T} 1, \mathrm{~T} 2$ & 40 & 1 & & \\
\hline $\mathrm{T} 3, \mathrm{~T} 4$ & 80 & 4.327 & & \\
\hline Lymph node metastasis & & & $1.074-3.486$ & 0.028 \\
\hline Absent & 50 & 1 & & \\
\hline Present & 70 & 1.935 & & \\
\hline miR-1260b expression & & & $1.191-3.781$ & 0.011 \\
\hline Low & 53 & 1 & & \\
\hline High & 67 & 2.122 & & \\
\hline
\end{tabular}

size, degree of differentiation, depth of invasion, or tumor location $(P>0.05)$.

\section{miR-1260b expression and CRC patient prognosis}

In order to analyze the relationship of miR-1260b expression levels between and prognosis of patient with CRCs, we plotted the survival curves (DFS and OS) of all patients by KaplanMeier method. The results suggested that the CRC patients with high miR-1260b expression levels had significantly shorter survival rates than low miR-1260b expression patients. (Figure 3). Univariate analysis showed that venous invasion $(P<0.05)$, depth of invasion $(P<0.001)$, lymph node metastasis $(P<0.001)$, and high miR-1260b expression levels $(P=0.003)$ were significantly correlated with OS (Table 2). Multivariate analysis using the Cox regression model suggested that high miR-1260b expression was an independent indicator of poor patient prognosis (Table 3).

\section{Discussion}

At present there are few reports about miR-1260b and there is no published research on the correlation between CRC and miR-1260b. Earlier studies showed that miR-1260b was abnormally and highly expressed in human peripheral blood dendritic cells, which suggests it may play an important role in the immune response of dendritic cells [12]. Stoecklin et al. reported that miR-1260b may be associated with gum disease [13]. In addition, miR-1260b is a molecular marker with high sensitivity and specificity. Hirata et al. [14] determined that miR$1260 \mathrm{~b}$ was highly expressed in renal cell carcinoma; they found that miR-1260b promoted renal cancer cell proliferation and invasion in renal cell carcinoma cells and genistein inhibited
Wnt-signaling by regulating miR-1260b expression in renal cancer cells. Another study reported that genistein exerts its anti-tumor effect via downregulation of miR-1260b, which target sRRP1 and Smad4 genes in prostate cancer cells4[15]. The expression level of miR-1260b in non-small cell lung cancer tissues was higher than that of adjacent tissues, which might be related to the development of non-small cell lung cancer [16]. Therefore, miR-1260b may be involved in a variety of cellular activities, which provides a new way of thinking and direction for the study of CRC.

The existing research shows many miRNAs promote or inhibit the development of CRC through a variety of ways, and that miRNAs have phase specificity during tumor development $[17,18]$. Particularly, the same tumor has different miRNA expression profiles in different stages of development $[19,20]$. In a recent study, miRNAs were routinely monitored to predict onset of metastasis in breast cancer. Madhavan et al. identified panels of miRNAs, which include the metastasis-promoting miRNAs family, as well as oncogenic and tumor-suppressive miRNAs, which can serve as prognostic markers for malignant breast tumors. These studies provide us with a new way explore the panel of multiple miRNAs as prognostic factor of CRC [21]. In our study, we discovered that the expression levels of miR-1260b increased in CRC tissues compared with para-carcinoma tissues, and the miR-1260b expression was also up-regulated in CRC tissues with positive lymph nodes. We also evaluated the relationship between the expression level of miR-1260b and clinicopathological features of patients with CRC. The results showed that high miR-1260b expression was closely related with lymph node metastasis and venous invasion, which means that miR-1260b promotes the early-stage metastasis of CRC. In addition, we plotted the curves of DFS and OS using the Kaplan-Meier method, showing that higher 
expression level of miR-1260b was closely related with poor DFS and OS, suggesting that miR-1260b may be a poor marker for prognosis of patients with CRC. Moreover, we used Cox regression analysis to evaluate some clinical and pathological factors. The final results suggest that high miR-1260b expression is an independent indicator of poor patient prognosis.

\section{Conclusions}

The high expression of miR-1260b suggests poor prognosis in patients with CRC. The results from this study conclude that

\section{References:}

1. Hoffmeister M, Jansen L, Stock C et al: Smoking, lower gastrointestinal endoscopy, and risk for colorectal cancer. Cancer Epidemiol Biomarkers Prev. 2014; 23(3): 525-33

2. Kraus S, Nabiochtchikov I, Shapira S, Arber N. Recent advances in personalized colorectal cancer research. Cancer Lett, 2014; 347(1): 15-21

3. Shao DD, Xue W, Krall EB et al: KRAS and YAP1 converge to regulate EMT and tumor survival. Cell, 2014; 158(1): 171-84

4. Thomas J, Ohtsuka M, Pichler M, Ling H: MicroRNAs: Clinical Relevance in Colorectal Cancer. Int J Mol Sci, 2015; 16(12): 28063-76

5. Ganci F, Vico C, Korita E et al: MicroRNA expression profiling of thymic epithelial tumors. Lung Cancer, 2014; 85(2): 197-204

6. Cherni I, Weiss GJ: miRNAs in lung cancer: Large roles for small players. Future Oncol, 2011; 7(9): 1045-55

7. Le Large TY, Meijer LL, Mato Prado $M$ et al: Circulating microRNAs as diagnostic biomarkers for pancreatic cancer. Expert Rev Mol Diagn, 2015; 15 $1-5$

8. Vidal DO, Marques MM, Lopes LF, Reis RM: The role of microRNAs in medulloblastoma. Pediatr Hematol Oncol, 2013; 30(5): 367-78

9. Ferracin $M$, Veronese A, Negrini M: Micromarkers: miRNAs in cancer diagnosis and prognosis. Expert Rev Mol Diagn, 2010; 10(3): 297-308

10. Hernando E: microRNAs and cancer: Role in tumorigenesis, patient classification and therapy. Clin Transl Oncol, 2007; 9(3): 155-60
miR-1260b may be an important molecular marker for the prognosis of CRC. However, more rigorous clinical and basic studies are needed to confirm the present results.

\section{Conflicts of interest}

The authors report no conflicts of interest in this work.

\section{Acknowledgement}

We thank all the people and patients who participated in this study

11. Pfaffl MW: A new mathematical model for relative quantification in realtime RT-PCR. Nucleic Acids Res, 2001; 29(9): e45

12. Jing Li, Liqin Li, Limin X et al: Expression of microRNA-1260b in non-smallcell lung carcinoma and the clinical significance. Chin J Exp Surg, 2015; 32(3): 624-26

13. Stoecklin-Wasmer C, Guarnieri P, Celenti R et al: MicroRNAs and their target genes in gingival tissues. J Dent Res, 2012; 91(10): 934-40

14. Hirata $\mathrm{H}$, Ueno $\mathrm{K}$, Nakajima $\mathrm{K}$ et al: Genistein downregulates onco-miR$1260 \mathrm{~b}$ and inhibits Wnt-signalling in renal cancer cells. Br J Cancer, 2013, 108(10): 2070-78

15. Hirata H, Hinoda $Y$, Shahryari $V$ et al: Genistein downregulates onco-miR$1260 \mathrm{~b}$ and upregulates SFRP1 and Smad4 via demethylation and histone modification in prostate cancer cells. Br J Cancer, 2014; 110(6): 1645-54

16. Xu L, Li L, Li J et al: Overexpression of miR-1260b in non-small cell lung cancer is associated with lymph node metastasis. Aging Dis, 2015; 6(6): $478-85$

17. Ye JJ, Cao J: MicroRNAs in colorectal cancer as markers and targets: Recent advances. World J Gastroenterol, 2014; 20(15): 4288-99

18. Watanabe T: Biomarker for high-risk patients with stage II colon cancer Lancet Oncol, 2013; 14(13): 1247-48

19. Ohtsuka $M$, Ling $H$, Doki $Y$ et al: MicroRNA processing and human cancer. J Clin Med, 2015; 4(8): 1651-67

20. Song SJ, Poliseno L, Song MS et al: MicroRNA-antagonism regulates breast cancer stemness and metastasis via TET-family-dependent chromatin remodeling. Cell, 2013; 154(2): 311-24 